\title{
On the wave solutions to the TRLW equation
}

\author{
Tukur Abdulkadir Sulaiman ${ }^{1,2,}$ Canan Unlu*, and Hasan Bulut ${ }^{1,4}$ \\ ${ }^{1}$ Department of Mathematics, Firat University, Elazig, Turkey \\ ${ }^{2}$ Department of Mathematics, Federal University Dutse, Jigawa, Nigeria \\ ${ }^{3}$ Department of Mathematics, Istanbul University, Istanbul, Turkey \\ ${ }^{4}$ Department of Mathematics Education, Final University, Girne, Cyprus
}

\begin{abstract}
In this study, a nonlinear model is investigated, namely; the time regularized long wave equation. Various solitary wave solutions are constructed such as the nontopological, compound topological-non-topological bell-type, singular and compound singular soliton solutions. Under the choice of suitable parameters values, the $2 \mathrm{D}$ and $3 \mathrm{D}$ graphs to all the obtained solutions are plotted. The reported results in this study may be helpful in explaining the physical meanings of some important nonlinear models arising in the field of nonlinear science.
\end{abstract}

\section{Introduction}

For the past two decades, the investigation of the solutions to the various kind of nonlinear evolution equations (NLEEs) has attracted the attention of many researchers. Nonlinear evolution equations are often used to model various nonlinear complex physical aspects that arise in the various fields on nonlinear physical sciences, especially in physics plasma, biology and fluid mechanics and so on. Various analytical techniques have been used to explore search of several NLEEs [1-11].

However, in the present study, the extended sinh-Gordon equation expansion method [12-15] (ShGEEM) will be used to investigate the wave solutions to the time regularized long wave (TRLW) equation [16]. The time regularized long wave is given by

$$
u_{t}+u_{x}+\beta u u_{x}+u_{x t t}=0
$$

where $u(x, t)$ is the unknown function of $x$; the spatial coordinate and the time $t$, respectively, and $\beta$ is a nonzero constant.

\section{Application}

In this section, the application of the extended sinh-Gordon equation expansion method to the TRLW is presented.

Consider the travelling wave transformation

*Corresponding Author: unlucanan@hotmail.com 


$$
u=\Psi(\eta), \eta=\mu(x-v t) .
$$

Inserting Eq. (2.1) into Eq. (1.1), yields

$$
2 \mu^{2} v^{2} \Psi^{\prime \prime}+2(1-v) \Psi+\beta \Psi^{2}=0
$$

By the extended ShGEEM [12], the solutions of any given nonlinear partial differential equation are assumed to be of the forms

$$
\begin{aligned}
& \Psi(w)=\sum_{j=1}^{m}\left[B_{j} \sinh (\theta)+A_{j} \cosh (w)\right] \cosh ^{j-1}(w)+A_{0}, \\
& \Psi(\eta)=\sum_{j=1}^{m}\left[ \pm i B_{j} \operatorname{sech}(\eta) \pm A_{j} \tanh (\eta)\right] \tanh ^{j-1}(\eta)+A_{0}, \\
& \Psi(\eta)=\sum_{j=1}^{m}\left[ \pm B_{j} \operatorname{csch}(\eta) \pm A_{j} \operatorname{coth}(\eta)\right] \operatorname{coth}^{j-1}(\eta)+A_{0},
\end{aligned}
$$

where $i=\sqrt{-1}$ and $w^{\prime}=\sinh (w)[5]$

Balancing the terms $\Psi^{\prime \prime}$ and $\Psi^{2}$, yields $m=2$.

With $m=2$, Eqs. (2.3), (2.4) and (2.5) take the forms

$$
\Psi(w)=B_{1} \sinh (w)+A_{1} \cosh (w)+B_{2} \cosh (w) \sinh (w)+\mathrm{A}_{2} \cosh ^{2}(w)+A_{0}(2.6)
$$

Inserting Eq. (2.6) and its second derivative into Eq. (2.2), gives a polynomial in powers of hyperbolic functions. Summing each coefficients of the hyperbolic functions of the same degree and equating each summation to zero, gives a group of algebraic equations. The group of algebraic equations is then simplified to secure the values of the parameters involved into different cases. For each case, inserting the obtained values of the parameters into each of Eqs. (2.4) and (2.5) with $m=$ 2, yields the solutions to Eq. (1.1).

\section{Case-1: When}

$$
A_{0}=\frac{6(-1+v)}{\beta}, A_{1}=0, B_{1}=0, A_{2}=\frac{6-6 v}{\beta}, B_{2}=-\frac{6 \sqrt{(-1+v)^{2}}}{\beta}, \mu=-\frac{\sqrt{-1+v}}{v},
$$

we get 
$u_{1}(x, t)=\frac{1}{\beta}\left(6(-1+v) \operatorname{sech}\left[\frac{\sqrt{-1+v}(x-t v)}{v}\right]^{2}+6 \ddot{z}(v-1) \operatorname{sech}\left[\frac{\sqrt{-1+v}(x-t v)}{v}\right] \tanh \left[\frac{\sqrt{-1+v}(x-t v)}{v}\right]\right)(2.7)$

$u_{2}(x, t)=-\frac{6 \sqrt{(-1+v)^{2}} \operatorname{coth}\left[\frac{\sqrt{-1+v}(x-t v)}{v}\right] \operatorname{csch}\left[\frac{\sqrt{-1+v}(x-t v)}{v}\right]+6(-1+v) \operatorname{csch}\left[\frac{\sqrt{-1+v}(x-t v)}{v}\right]^{2}}{\beta}(2.8)$

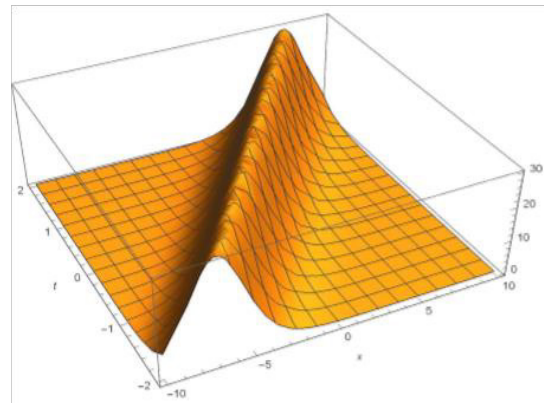

(a)

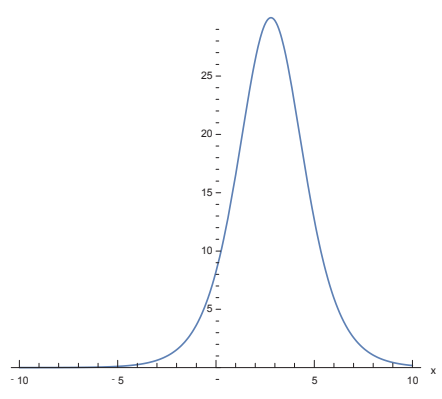

(b)

Figure 1: The 3D and 2D graphs of the real part of Eq. (2.7).

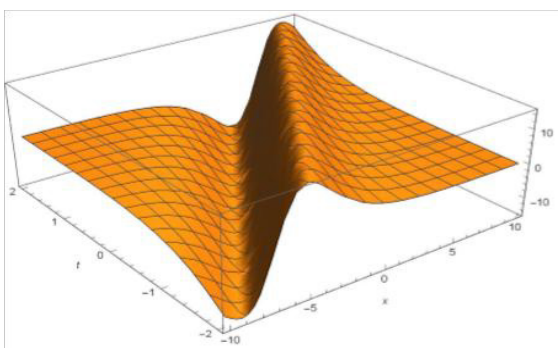

(a)

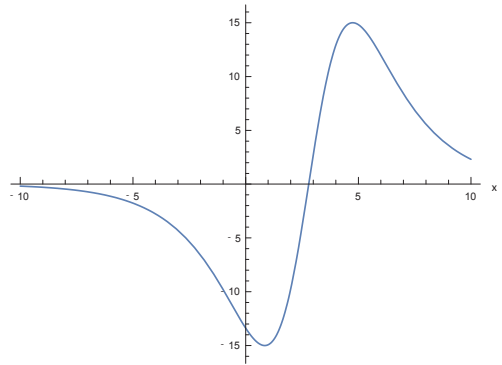

(b)

Figure 2: The 3D and 2D graphs of the imaginary part of Eq. (2.7).

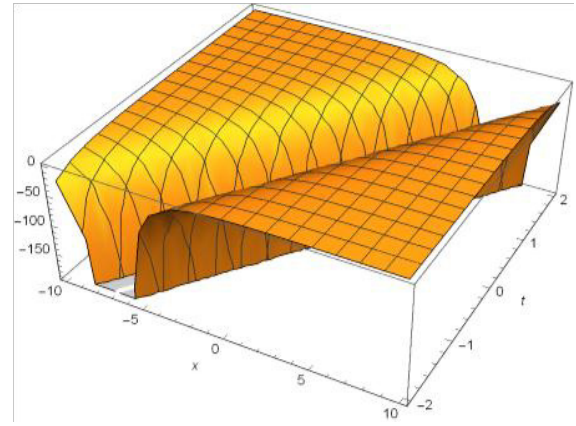

(a)

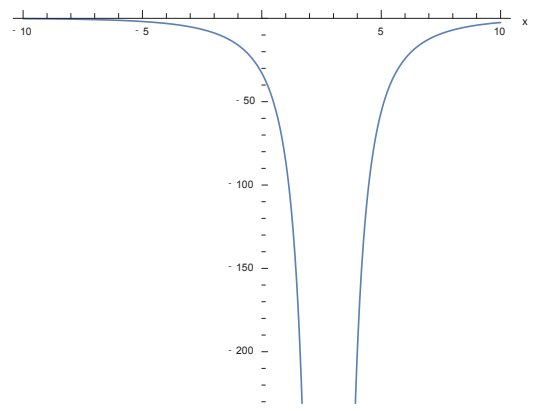

(b) 
Figure 3: The 3D and 2D graphs of Eq. (2.8).

\section{Case-2: When}

$$
A_{0}=\frac{1-v}{\beta}, A_{1}=0, B_{1}=0, A_{2}=\frac{3(-1+v)}{\beta}, B_{2}=0, \mu=\frac{\sqrt{-(v-1)}}{2 v},
$$

we get

$$
u_{3}(x, t)=\frac{(-1+v)\left(-2+\cosh \left[\frac{\sqrt{1-v}(x-t v)}{v}\right]\right) \operatorname{sech}^{2}\left[\frac{\sqrt{1-v}(x-t v)}{v}\right]}{\beta}
$$

And

$u_{4}(x, t)=\frac{(-1+v)\left(2+\operatorname{Cosh}\left[\frac{\sqrt{1-v}(x-t v)}{v}\right]\right) \operatorname{csch}^{2}\left[\frac{\sqrt{1-v}(x-t v)}{v}\right]}{\beta}$

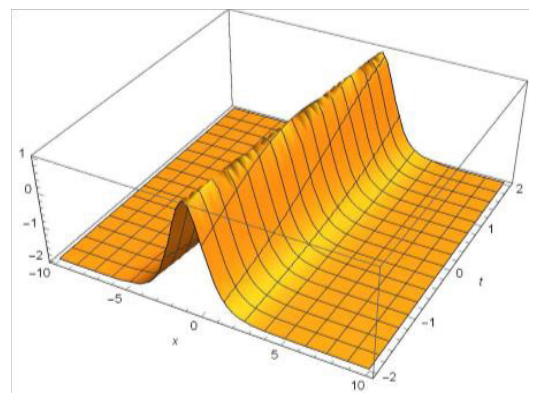

(a)

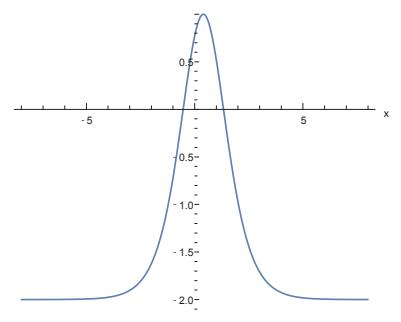

(b)

Figure 3: The 3D and 2D graphs of Eq. (2.9).

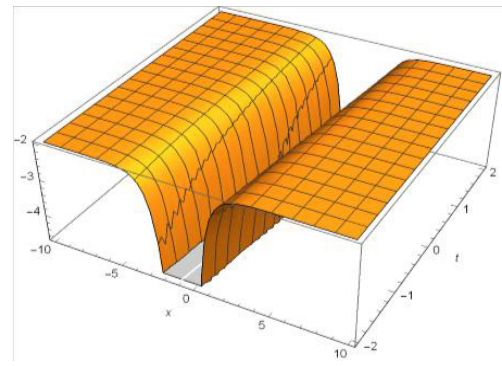

(a)

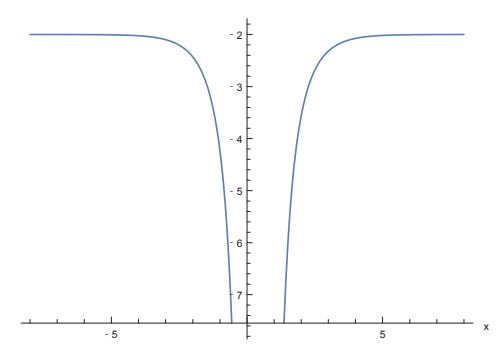

(b)

Figure 4: The 3D and 2D graphs of Eq. (2.10). 


\section{Conclusion}

In this study, the time regularized long wave equation is investigated by using the extended sinhGordon equation expansion method with the aid of Wolfram Mathematica 11. Various soliton solutions such as the non-topological, compound topological, non-topological bell-type, singular and compound singular solitons are successfully extracted. Using suitable values of parameters, the $2 \mathrm{D}$ and $3 \mathrm{D}$ graphics to all the reported solutions are plotted.

\section{References}

1. Z. Yan, MM Research Preprints, 22 363-375 (2003)

2. H. Bulut, T.A. Sulaiman and H.M. Baskonus, Opt Quant Electron, 48564 (2016)

3. H. Bulut, T.A. Sulaiman, H.M. Baskonus and A.A. Sandulyak, Optik, 135 327-336 (2017)

4. Md. Nur Alam, F.M. Belgacem and M.A. Akbar, Journal of Applied Mathematics and Physics, 3 1571-1579 (2015)

5. H. Bulut, H.A. Isik, T.A. Sulaiman, ITM Web of conferences, 13, 01019 (2017)

6. S. Duran, M. Askin, T.A. Sulaiman, IJOCTA, 7(3), 240-247 (2017)

7. H. Bulut, T.A. Sulaiman, H.M. Baskonus, Eur. Phys. J. Plus, 132, 459 (2017)

8. H.M. Baskonus, H. Bulut, T.A. Sulaiman, Eur. Phys. J. Plus, 132, 482 (2017)

9. H. Bulut, T.A. Sulaiman, B. Demirdag, Nonlinear Dyn, 91, 1985-1991 (2018)

10. T.A. Sulaiman, T. Akturk, H. Bulut, H.M. Baskonus, Journal of Electromagnetic Waves and Applications, 32(9) 1093-1105 (2017)

11. A. Yokus, T.A. Sulaiman, H. Bulut, Opt Quant Electron, 5031 (2018)

12. H. Bulut, T.A. Sulaiman and H.M. Baskonus, Optik, 163 1-7 (2018)

13. H.M. Baskonus, T.A. Sulaiman, H. Bulut, Opt Quant Electron, 50, 14 (2018)

14. C. Cattani, T.A. Sulaiman, H.M. Baskonus andH. Bulut, Eur. Phys. J. Plus 133, 228 (2018)

15. C. Cattani, T.A. Sulaiman, H.M. Baskonus and H.Bulut, 50, Opt Quant Electron 138 (2018)

16. K.S. Hossain, M.A. Akbar and Md.A.K. Azad, Cogent Physics, 4396948 (2017) 